\title{
Método Deming y los procesos de explotación
}

\author{
Deming Management Method and Exploitation Processes
}

\author{
Luis Lemaitre Mostajo \\ llemaitre93@outlook.com \\ Código ORCID: 0000-0003-3260-9809 \\ Banco Fassil Sociedad Anónima \\ Santa Cruz - Bolivia
}

Artículo recibido junio 2020 / Arbitrado en octubre 2020 / Aceptado en octubre 2020 / Publicado en enero 2021

RESUMEN

El objetivo de la investigación fue diseñar una estrategia que permita optimizar los procesos de explotación, utilizando la información generada por un sistema de planificación de recursos empresariales como soporte para la toma de decisiones; se desarrolló bajo un enfoque mixto de corte transversal y alcance descriptivo. El objeto de estudio fue una empresa con 32 trabajadores que se dedica a la extracción de yeso en Bolivia, se aplicó un censo a los trabajadores para analizar la percepción sobre los beneficios tras tres años de vigencia del sistema informático; asimismo, se estudiaron los estados financieros de cinco gestiones. Como resultado se comprobó la existencia de beneficios tangibles obtenidos gracias a la implementación del sistema informático y se propuso una estrategia de mejora continua basada en el método Deming. Se concluyó que los sistemas informáticos se constituyen en herramientas eficientes para la toma de decisiones y deben aplicarse acompañadas a otras herramientas de mejor continua.

Palabras clave: Procesos; Optimización; Industria; Planificación; Recursos; Yeso

ABSTRACT The objective of the research was to design an optimization strategy for exploitation processes, using the information generated by an enterprise resource planning system as support for decision-making; It was developed under a mixed cross-sectional approach and descriptive scope. The object of study was a Bolivian company with 32 workers that is dedicated to the extraction of plaster, a census was applied to the workers to analyze the perception of the benefits after three years of use of the computer system; Likewise, the financial statements of five administrations were studied. As a result, the existence of tangible benefits obtained thanks to the implementation of the computer system was verified and a continuous improvement strategy based on the Deming method was proposed. It was concluded that computer systems constitute efficient tools for decisionmaking and should be applied together with continuous improvement tools.

Key words: Processes; Optimization; Industry; Planning; Resources; Plaster 
INTRODUCCIÓN

La toma de decisiones empresariales exige cada vez mayor rapidez y precisión, la competitividad y eficiencia no son negociables. Las organizaciones que no se adapten a la tecnología de soporte para mejorar continuamente los procesos, podrán correr el riesgo de perder espacio en el mercado. Las tecnologías de la información se han convertido en herramientas cada vez más valiosas, jugando un papel fundamental en los cambios de los paradigmas de cómo se hacen los negocios; convirtiéndose así, en una necesidad.

En este sentido, resulta importante tomar información de cada sistema y concentrarla en un informe, para tomar decisiones a nivel gerencial. Existe una amplia gama de programas que aporten herramientas de control para el mejoramiento de procesos internos, aportando mayor confiabilidad, mejorando el tiempo de repuesta a diversos problemas, disminuyendo costos, incrementando utilidades y disminuyendo la burocracia.

En los años 90 aparece el software ERP (Enterprise Resource Planning) que en español se conoce como sistema de planificación de los recursos empresariales, consiste en un conjunto de aplicaciones integradas capaces de unir todas las transacciones internas de una organización (Uwizeyemungu \& Raymond, 2010). Actualmente existen proveedores con presencia a nivel mundial; el Software más reconocido es SAP (Systeme, Andwendungen, Produkte) y es usado por empresas de gran tamaño (Pollock \& Williams, 2008). Para las empresas pequeñas y medianas existen sistemas más adecuados, económicos e inclusive gratuitos (Pinto et al., 2017). En términos generales los sistemas ERP facilitan el flujo de información a través de las distintas áreas de una empresa, permitiendo que los datos sean compartidos y procesados de manera intercambiable y mejorando la gestión de los procesos de negocio (Azzarini et al., 2011).

Por otra parte, existen diferentes herramientas diseñadas para apoyar la toma de decisiones empresariales, algunas de diseño, otras de estrategia. El círculo de Deming se constituye en una herramienta que describe los cuatro pasos esenciales que se deben llevar a cabo de forma sistemática para lograr la mejora continua (Walton, 1988).

En los países en desarrollo, las empresas no siempre logran aplicar los sistemas y herramientas previamente señalados, lo que conduce a baja calidad de los productos y servicios producidos, gran cantidad de fallos en los procesos productivos, baja eficacia y eficiencia, problemas de control, desconocimiento de riesgos potenciales, entre otros (Díaz et al.,2005). En estas empresas, cuando se piensa en los recursos, se suele considerar capital, activos y personal, olvidando muchas veces que la información generada es invaluable y el modo de gestionarla puede determinar el éxito o fracaso de una gestión.

Si bien no todos los sistemas ERP son costosos, su proceso de adopción está a menudo relacionado con riesgos; si falla la implementación o post implementación, puede ocasionar grandes pérdidas para las empresas.

Este estudio consideró el caso de una empresa boliviana dedicada a la extracción de yeso, con tres años de experiencia en la implementación de un sistema ERP, con base en Software libre diseñado a medida. El objetivo de la investigación fue diseñar la estrategia que permita optimizar los procesos 
de explotación de yeso, utilizando la información generada por el Sistema ERP como soporte para la toma de decisiones. Para alcanzar el objetivo, la investigación se constituyó en tres etapas: percepción del impacto del sistema ERP, análisis de los indicadores financieros y aplicación del método Deming en una empresa explotadora de yeso.

\section{METODOLOGÍA}

La investigación se desarrolló bajo un enfoque mixto (cuantitativo y cualitativo), de corte transversal y alcance descriptivo; se logró diseñar una estrategia que permita contar con un proceso de explotación de yeso eficiente, utilizando la información generada por el Sistema ERP, siguiendo un proceso de tres etapas: (1) Análisis de la percepción del impacto del sistema ERP; (2) Análisis de los indicadores financieros, tomando cinco gestiones; (3) Aplicación del método Deming para el diseño de la estrategia.

En la primera etapa se aplicó la técnica del censo y la entrevista, tomando como población objetivo a los trabajadores de una empresa extractora de yeso. La boleta censal y la guía de la entrevista fueron diseñadas para conocer su percepción sobre los efectos positivos y negativos de la implementación generada por el Sistema ERP; así como para determinar las expectativas que tiene a futuro sobre su utilidad. Ambos instrumentos fueron validados por criterio de expertos en consultoría empresarial para el rediseño de procesos industriales.

En la segunda etapa se aplicó el método histórico lógico, mismo que permitió la revisión y el análisis de la evolución de indicadores financieros de la empresa. Los indicadores considerados fueron los siguientes: liquidez corriente, prueba ácida, rotación de inventarios, rotación de activos, rotación de cuentas por cobrar, razón de endeudamiento, ratio de solvencia, apalancamiento financiero, ratio a corto plazo, ratio de largo plazo, margen de ganancia bruta, margen de ganancia neta, ROI y ROE. Se tuvo acceso a los estados financieros de cinco gestiones, de las cuales los tres últimos años representan el periodo post implementación.

La tercera estuvo orientada al diseño de la estrategia de optimización del proceso de explotación; se aplicó el método analítico sintético y el método de Deming. El libro consultado para aplicar este último método fue el presentado por Deming, \& Medina (1989). Asimismo, se consultaron los aportes de Vera (2006), Knouse et al. (2009), Díaz et al. (2005) y Walton, (1988).

\section{RESULTADOS}

A continuación, se presentan los resultados de la investigación, en tres etapas: Percepción del impacto del sistema ERP, análisis de los indicadores financieros y aplicación del método Deming en una empresa explotadora de yeso.

Análisis de la percepción del imparto del Sistema ERP

Se determinaron las áreas de la Empresa afectadas por la implementación del Sistema ERP y el efecto; mediante una correlación entre los indicadores de gestión y los efectos percibidos, por personal de nivel gerencial y operativo. Los resultados se presentan en la Tabla 1. 


\section{Tabla 1}

Sistemas ERP - Impacto percibido e impacto potencial, áreas explotación y contabilidad

\begin{tabular}{|c|c|c|c|}
\hline Efectos & Factores & $\begin{array}{c}\text { Impacto } \\
\text { Percibido } \\
\%\end{array}$ & $\begin{array}{c}\text { Impacto } \\
\text { Potencial } \\
\%\end{array}$ \\
\hline \multirow{5}{*}{ Operacionales } & Reducción de tiempos operativos & $27 \%$ & $24 \%$ \\
\hline & Eliminación de procesos repetitivos & $29 \%$ & $24 \%$ \\
\hline & Reducción de inventarios & $21 \%$ & $22 \%$ \\
\hline & Dependencia tecnológica & $15 \%$ & $16 \%$ \\
\hline & Aumento de la carga de trabajo & $8 \%$ & $14 \%$ \\
\hline \multirow{4}{*}{ Gestión } & Totales & $100 \%$ & $100 \%$ \\
\hline & Mejor planificación para el uso de recursos & $40 \%$ & $35 \%$ \\
\hline & Estructura de procesos administrativos & $33 \%$ & $35 \%$ \\
\hline & Aumento de la gestión documental & $28 \%$ & $30 \%$ \\
\hline \multirow{5}{*}{ Infraestructura } & Totales & $100 \%$ & $100 \%$ \\
\hline & Visibilidad de la información para toda la empresa & $47 \%$ & $39 \%$ \\
\hline & Construcción de objetivos comunes & $36 \%$ & $36 \%$ \\
\hline & Costos de mantenimiento & $17 \%$ & $25 \%$ \\
\hline & Totales & $100 \%$ & $100 \%$ \\
\hline \multirow{5}{*}{ Organizacional } & $\begin{array}{l}\text { Aumento de la capacidad de procesamiento de la } \\
\text { información }\end{array}$ & $36 \%$ & $28 \%$ \\
\hline & Reducción de herramientas tecnológicas & $23 \%$ & $26 \%$ \\
\hline & Comprensión del funcionamiento global de la Empresa & $22 \%$ & $26 \%$ \\
\hline & Cambio en los procedimientos de trabajo & $19 \%$ & $19 \%$ \\
\hline & Totales & $100 \%$ & $100 \%$ \\
\hline
\end{tabular}

La Tabla 1 analiza el peso ponderado de los efectos percibidos y potenciales del sistema $E R P$, según el criterio de los trabajadores a nivel estratégico y operativo, en áreas de explotación y contabilidad. Los resultados presentados en la primera columna (impacto percibido) permiten valorar en qué factores puntuales se ha recibido cambios y su peso ponderado dentro del área. Los resultados presentados en la segunda columna (impacto esperado) permiten valorar en qué factores puntuales se espera cambios a futuro y su peso ponderado dentro de cada área. Estos resultados no discriminan si los efectos son positivos o negativos.
Por este motivo se presenta la ecuación 1 , que muestra la relación entre impactos positivos e impactos totales (recibidos y esperados).

Ecuación 1: Impacto percibido del Sistema ERP

Relación positiva $=$ Impacto positivo $/$ Impacto total $=82 / 100=0,82=82 \%(1)$

El resultado de la ecuación 1 muestra que para los trabajadores de áreas explotación y contabilidad, la implementación del Sistema ERP tuvo un impacto positivo importante en el desempeño y desarrollo de sus actividades. Se presenta un análisis detallado para cada efecto identificado (ver figuras 1-4). 


\section{Figura 1}

Efectos operacionales causados por el Sistema ERP

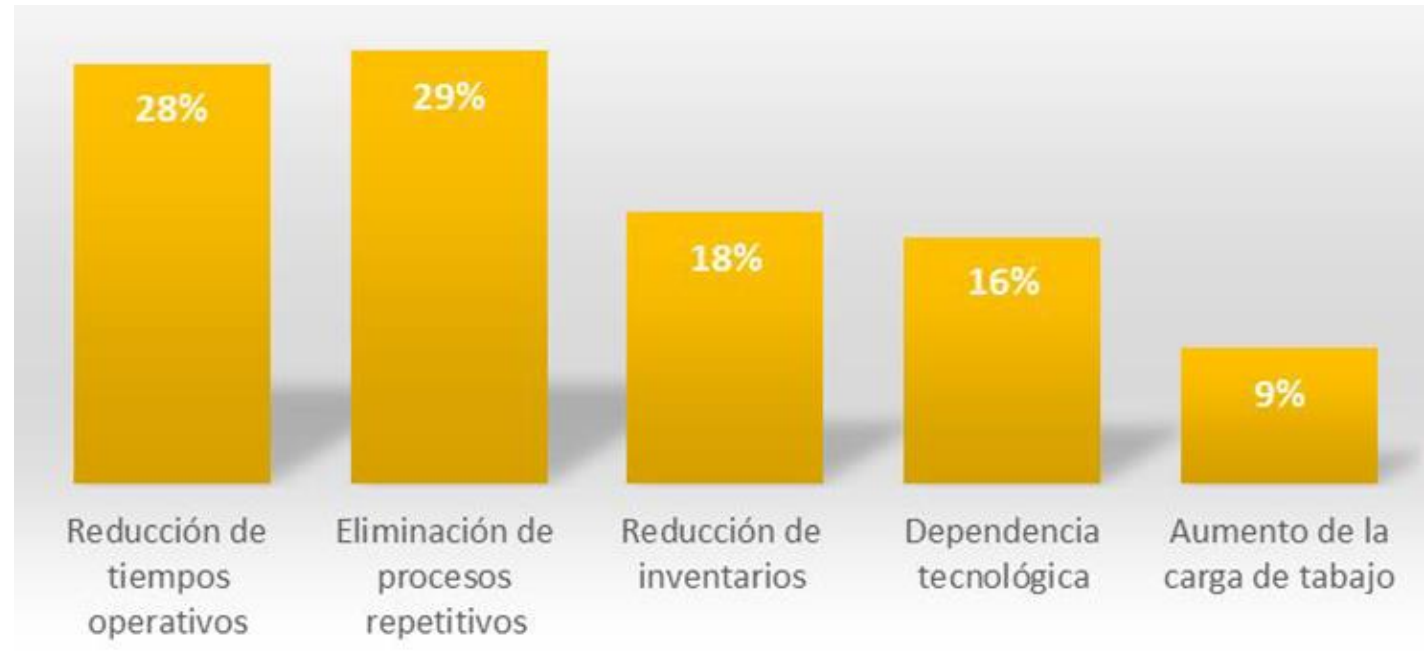

En la figura 1, de manera general, los funcionarios afirman que la integración es útil; se observa que el factor que tuvo mayor impacto fue la eliminación de procesos repetitivos (29\%); otro aspecto que destacan es la reducción de tiempos operativos (28\%), ya que se puede contar con información en tiempo real que agiliza los procesos; informan que existe un mejor control y manejo de los materiales y recursos, reducción de inventarios (18\%); la dependencia tecnológica (16\%), es un aspecto que muestra un impacto bajo, la ven como una herramienta de soporte para el mejor manejo de la información; el aumento de la carga de trabajo (9\%), presenta un impacto bajo, argumentan su malestar indicando que ha sido un perjuicio.

\section{Figura 2}

Efectos de gestión causados por el Sistema ERP

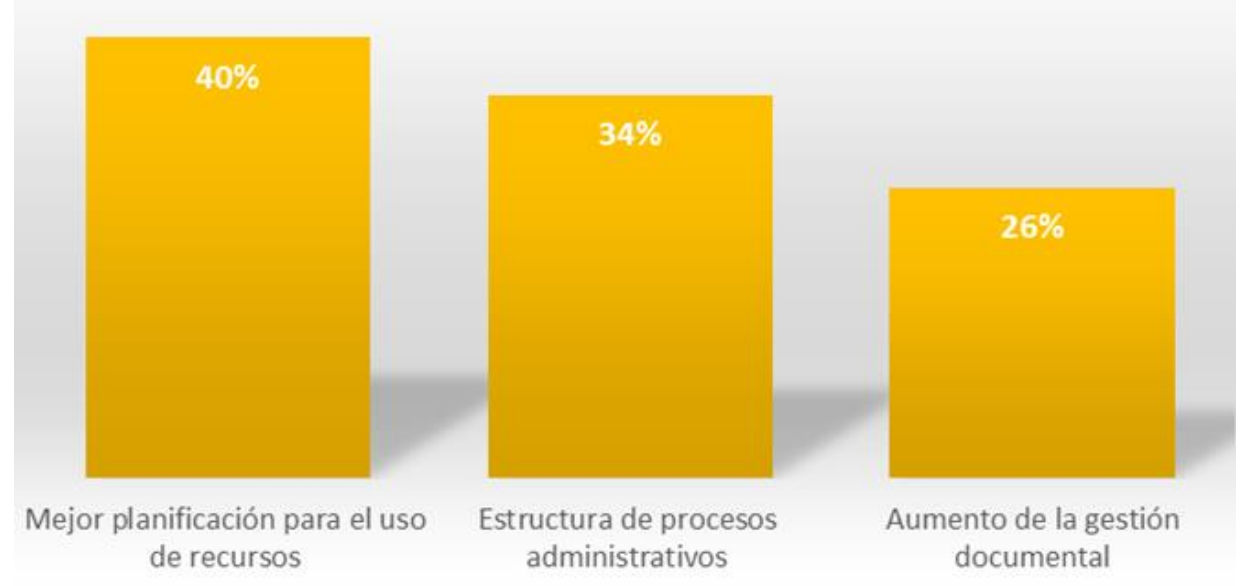


Cuando se hace referencia a los beneficios de gestión, según la Figura 2, se ve el efecto de mayor impacto para los trabajadores en la mejor planificación para el uso de los recursos (40\%), Los consultados argumentan que la empresa cuenta con información actualizada y oportuna, permitiendo mejor manejo de recursos; otro efecto es la estructuración de los procesos administrativos (34\%), se hizo una restructuración de los procesos para adaptar los módulos del sistema a la empresa; por último, el aumento de la gestión documental (26\%), que considera que los trámites (permisos de trabajo, solicitudes de materiales, información de clientes, etc.) se incrementaron, algunos trabajadores lo han asimilado como un perjuicio, y otros como algo positivo ya que a nivel gestión se necesita de este tipo de información para poder planificar estrategias gerenciales y organizacionales.

\section{Figura 3}

Efectos en infraestructura causados por el Sistema ERP

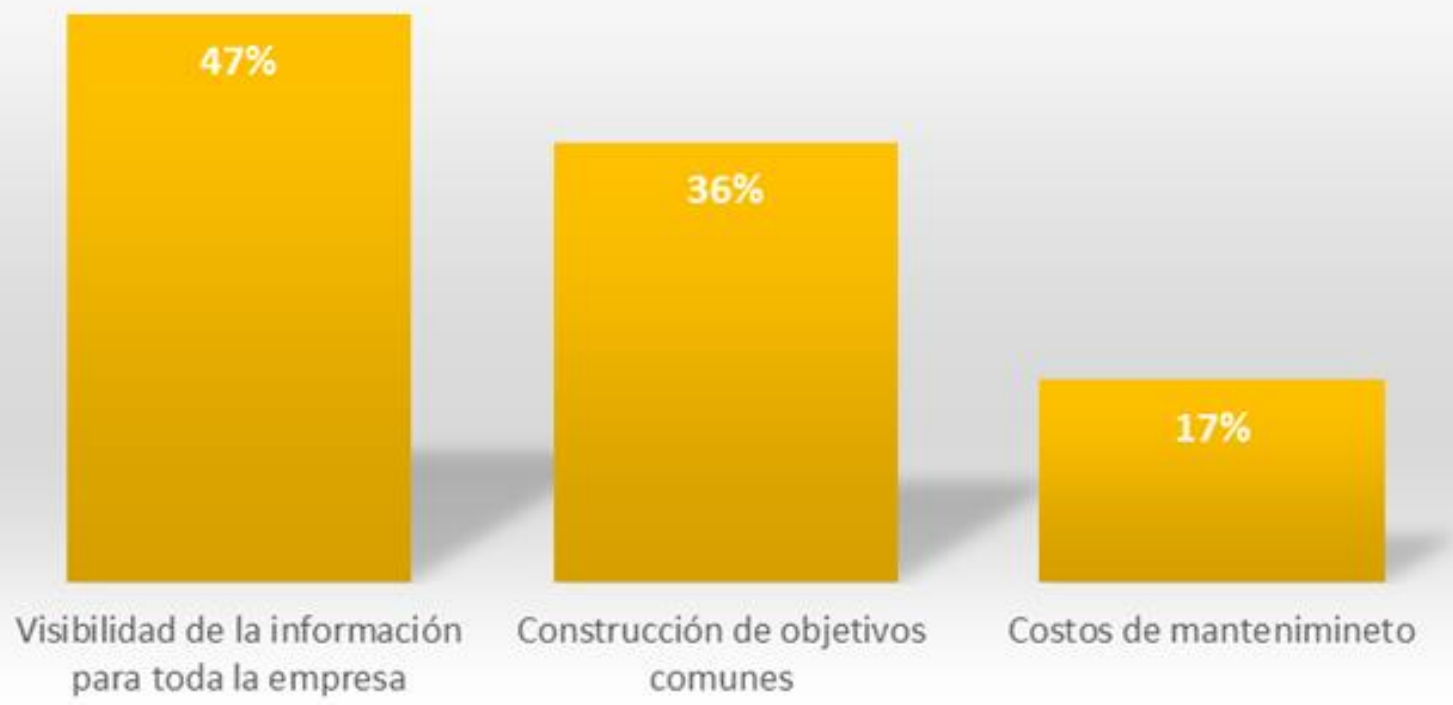

Como se observa en la Figura 3, al hacer referencia a los beneficios en Infraestructura, tuvo mayor impacto para los trabajadores la mayor visibilidad de la información para toda la empresa (47\%), siendo esta una de las principales características de los Sistemas ERP, debido a su carácter integrador. Los consultados argumentan que se eliminan procesos repetitivos haciéndolos más eficientes; los trabajadores percibieron un impacto importante en la construcción de objetivos comunes (36\%); y por último dentro de estos efectos están los costos de mantenimiento (17\%), los consultados expresan que los gastos no son elevados; sin embargo, algunos trabajadores expresan que no era necesario incrementar estos costos. 


\section{Figura 4}

Impactos organizacionales causados por el Sistema ERP

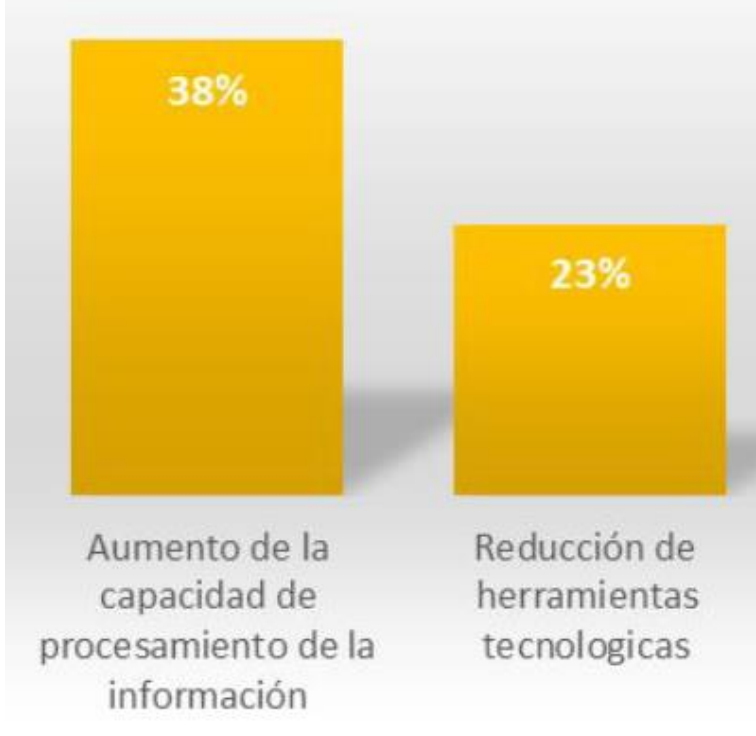

Según la Figura 4, dentro de los beneficios organizacionales destaca el aumento de la capacidad de procesamiento de la información (38\%), los funcionarios resaltan la capacidad del Sistema ERP de integrar la información de todas las áreas en un solo sistema con información actualizada y oportuna; en cuanto a la reducción de herramientas tecnológicas (23\%), la empresa sólo cuenta con un sistema en vez de tener uno para cada área; se tiene mayor comprensión del funcionamiento global (22\%), todos tienen acceso a la información; cambio en los procedimientos de trabajo
(18\%), los trabajadores no sintieron beneficio en estos cambios, inclusive algunos están inconformes por ello.

Análisis de financiero tras la implementación del Sistema ERP

Según la Tabla 2, estos resultados fueron contrastados con ratios financieros, haciendo un análisis histórico de dos gestiones antes de la implementación y tres gestiones posteriores a ésta. 


\section{Tabla 2}

Ratios financieros - antes y después de la implementación

\begin{tabular}{cccccc}
\hline \multirow{2}{*}{ RATIOS } & \multicolumn{3}{c}{ ANTES DEL ERP } & \multicolumn{3}{c}{ IMPLEMENTACION DEL ERP } \\
& Año $\mathbf{1}$ & Año 2 & Año 3 & Año 4 & Año 5 \\
\hline Liquidez corriente & 4,22 & 4,26 & $\mathbf{4 , 8 1}$ & $\mathbf{4 , 7 5}$ & $\mathbf{5 , 4 4}$ \\
Prueba ácida & 4,09 & 3,71 & $\mathbf{4 , 6 5}$ & $\mathbf{4 , 5 6}$ & $\mathbf{4 , 7 4}$ \\
Rotación de inventarios & 40,34 & 7,27 & $\mathbf{2 7 , 8 7}$ & $\mathbf{2 5 , 1 0}$ & $\mathbf{1 0 , 2 2}$ \\
Rotación de activos & 1,30 & 1,09 & $\mathbf{1 , 3 6}$ & $\mathbf{0 , 8 6}$ & $\mathbf{0 , 9 5}$ \\
Rotación de cuentas por cobrar & 9,25 & 3,83 & $\mathbf{6 , 0 2}$ & $\mathbf{6 , 9 6}$ & $\mathbf{8 , 2 3}$ \\
Razón de endeudamiento & 0,19 & 0,20 & $\mathbf{0 , 6 2}$ & $\mathbf{0 , 3 5}$ & $\mathbf{0 , 2 6}$ \\
Ratio de solvencia & 5,15 & 1,25 & $\mathbf{1 , 6 2}$ & $\mathbf{2 , 8 4}$ & $\mathbf{3 , 8 0}$ \\
Apalancamiento financiero & 1,24 & 1,25 & $\mathbf{1 , 0 0}$ & $\mathbf{1 , 5 4}$ & $\mathbf{1 , 3 6}$ \\
Ratio a corto plazo & 0,94 & 0,93 & $\mathbf{0 , 3 4}$ & $\mathbf{0 , 3 9}$ & $\mathbf{0 , 3 5}$ \\
Ratio de largo plazo & 0,06 & 0,07 & $\mathbf{0 , 6 6}$ & $\mathbf{0 , 6 1}$ & $\mathbf{0 , 6 5}$ \\
Margen de ganancia bruta & 0,26 & 0,32 & $\mathbf{0 , 2 9}$ & $\mathbf{0 , 2 2}$ & $\mathbf{0 , 3 1}$ \\
Margen de ganancia neta & 0,08 & 0,07 & $\mathbf{0 , 0 7}$ & $\mathbf{0 , 0 3}$ & $\mathbf{0 , 1 0}$ \\
ROI & 0,10 & 0,07 & $\mathbf{0 , 0 6}$ & $\mathbf{0 , 0 3}$ & $\mathbf{0 , 0 9}$ \\
ROE & 0,12 & 0,09 & $\mathbf{0 , 0 9}$ & $\mathbf{0 , 0 4}$ & $\mathbf{0 , 1 3}$ \\
\hline
\end{tabular}

Liquidez corriente, existe un aumento significativo desde el año 3 (gestión en la que se implementó el Sistema ERP), la empresa fue aumentando su capacidad de cumplir obligaciones.

Prueba ácida, los inventarios fueron aumentando desde la implementación del sistema, aunque en el transcurso de los años los porcentajes fueron disminuyendo con relación a otras cuentas de los activos. Los años 3 y 4 no fueron del todo eficientes, sin embargo, se observa un crecimiento con relación al año 2, gestión que tiene una disminución en la rotación. La empresa debe trabajar en este aspecto para mejorar la eficiencia en el manejo de los inventarios, haciendo un mejor uso de los recursos con los que cuentan para reducir costos de los minerales explotados.

Rotación de activos, indicador que demuestra un desempeño deficiente. Razón de endeudamiento, este indicador muestra un aumento de la dependencia de capital ajeno provocando un incremento acelerado del riesgo hasta el 2014, y a partir de ahí empieza a descender. en los años previos a la implementación, el porcentaje de financiamiento propio es mayor que el de los acreedores.

Rotación de cuentas por cobrar, la información obtenida muestra una tendencia a incrementar las veces que hace efectivos los cobros a clientes. El incremento coincide con la implementación del Sistema ERP.

Ratio de solvencia, desde la implementación del Sistema ERP cuenta con una mayor capacidad para hacer frente a estas obligaciones.

Apalancamiento financiero, en el periodo de análisis se observa una baja en el apalancamiento financiero en la gestión 2014, 
y a partir de ahí, empieza a ascender en las siguientes dos gestiones.

Ratio a corto plazo, mide el porcentaje correspondiente de las obligaciones a corto plazo con relación al total de las deudas; antes de la implementación del Sistema ERP el mayor porcentaje de la deuda era a corto plazo, lo que generaba mayores niveles de riesgo. Ratio a largo plazo, una vez implementado el Sistema ERP el porcentaje de la deuda a largo plazo respecto al total pasivo tiende a ser mayor porque la empresa cambia su estructura financiera, disminuyendo sus recursos provenientes de deudas de corto plazo.

Margen de ganancia bruta, este aspecto no resulta ser favorable, ya que los costos de producción tienden a aumentar, situación que empieza a cambiar en la gestión 2016 donde los costos bajan con relación al nivel de ventas, obteniendo mayor ganancia bruta, situación que se puede mejorar haciendo un mejor control del proceso de explotación de materias primas. Margen de ganancia neta, se alcanza un mayor porcentaje de ganancia respecto a las gestiones anteriores.

ROI, este indicador muestra un incremento considerable en la última gestión, denota una mayor eficiencia en el uso de inversión. ROE, el indicador muestra una variación positiva. Se tiene una mejor gestión de los procesos dentro de la Empresa.

Al contrastar la percepción de los trabajadores con los indicadores financieros se encontraron factores positivos y negativos que se analizan a continuación. Con relación a las fortalezas, se entiende que la sistematización de la información permite procesar la información de las diferentes áreas, provocando un mayor flujo de la información. Asimismo, el acceso a la información en tiempo real es altamente favorable tener un mejor manejo y control de los recursos, ha incrementado la eficiencia. Otra fortaleza identificada es la agilidad que provee para la toma de decisiones; al contar con información en tiempo real, permite tomar medidas correctivas oportunas. El Sistemas ERP ha sido elaborado según el tamaño y las necesidades de la empresa, bajo la legislación boliviana. Por último, el hecho de que el sistema sea gratuito (software libre), permite que la infraestructura tecnológica represente un costo bajo (500 dólares anuales).

Por otra parte, la principal debilidad es la estructura de costos de la empresa ya que no existe un tratamiento adecuado para asignar los costos indirectos a cada producto. Otro factor para analizar es la resistencia al cambio expresada por parte del personal, existe actitud negativa a cambiar su forma habitual de trabajar. La gerencia percibe necesidades de capacitación, que complican la adaptación a la automatización de procesos generando resistencia y rechazo a la implementación de la nueva tecnología; existe riesgo de que se ingrese incorrectamente los datos, ocasionando que la información no sea precisa al momento de tomar decisiones.

Es posible eliminar procesos repetitivos que están generando retraso y costos adicionales, dando la opción a desarrollar nuevas actividades e incursionar en nuevos mercados. Este sistema genera la posibilidad de acceder a una Certificación ISO 9001, ya que se tiene un mejor control y seguimiento de las actividades realizadas.

Los rápidos cambios tecnológicos hacen que las tecnologías requieran constantes cambios y actualizaciones, que resultan ser costosas. Se debe prever respuesta a posibles fallas de los servidores, por lo que se debe contar con planes de contingencias, por 
ejemplo, contar con redundancia de información (respaldos). Otra amenaza es la existencia de piratas tecnológicos que pueden vulnerar la seguridad del sistema y utilizar la información para fines ilegales. Por último, los factores climatológicos afectan la explotación del yeso, por lo que se necesita que el sistema ERP genere un control y seguimiento de explotación para tomar previsiones y cumplir metas.

\section{Estrategia para el mejoramiento continuo}

Se diseñó una estrategia de mejoramiento continuo que optimice el proceso de explotación de yeso, a partir del Sistema ERP; el Círculo de Deming, fue la herramienta que se ocupó para este efecto. El objetivo de la estrategia es optimizar los procesos de explotación de yeso, utilizando la información generada por el Sistema ERP como soporte para la toma de decisiones.

\section{Plan de mejoramiento continuo}

El proceso de explotación tiene tres áreas susceptibles de mejora que deben ser atendidas por la estrategia: insumos directos, mano de obra directa y costos indirectos. En la Figura 5 se presenta la estructura del plan de mejoramiento, se distinguen tres etapas. \}

\section{Figura 5}

Plan de mejoramiento continuo

\begin{tabular}{|c|}
\hline ETAPA 1 \\
\hline Establecer un Sistema de costeo por cantera \\
\hline Menor cantidad de desperdicios \\
\hline Reducir costos de produccion \\
\hline
\end{tabular}
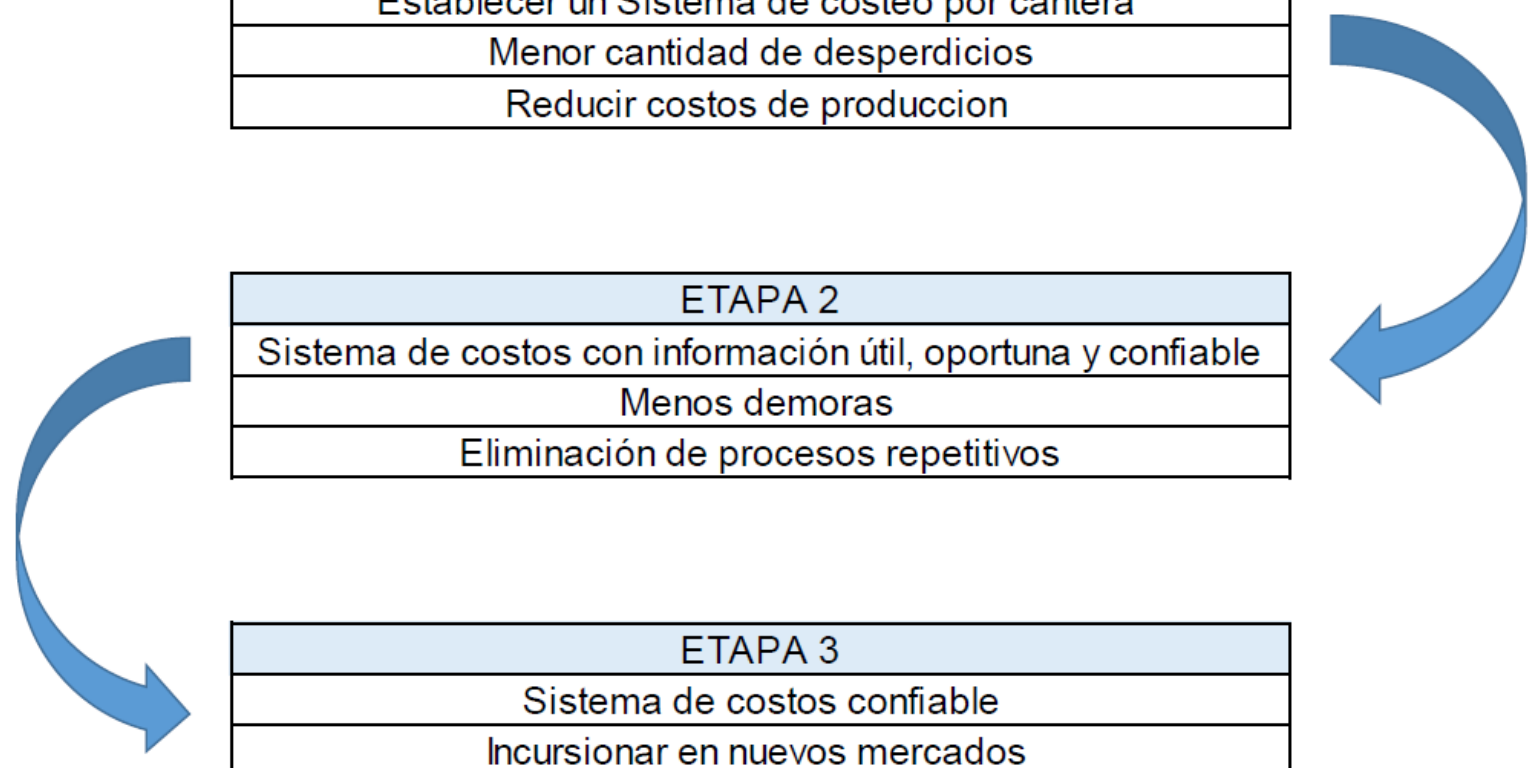

\section{ETAPA 2}

Sistema de costos con información útil, oportuna y confiable Menos demoras Eliminación de procesos repetitivos

\begin{tabular}{|c|}
\hline ETAPA 3 \\
\hline Sistema de costos confiable \\
\hline Incursionar en nuevos mercados \\
\hline Mejorar la calidad de los productos \\
\hline
\end{tabular}


Cada etapa de las mostradas en la Figura 3 constará de dos meses, en los que se esperan resultados específicos. En la primera etapa se pretende establecer un sistema de costo por cantera que reducirá los tiempos operativos al rediseñar el proceso de carguío de la materia prima, reduciendo el tiempo de horas/máquina y el uso de combustible. En la segunda etapa se contará con información de costos por cantera, así mismo se obtendrá menores demoras y se eliminarán procesos repetitivos que no generen valor. En la última etapa se presentará un sistema confiable de costos sobre los resultados de todo el proceso, se mejorará la productividad al aumentar la cantidad de materia prima explotada por jornada a un menor costo, lo que le permitirá incursionar en nuevos mercados al ofrecer un producto de mayor calidad a un precio más competitivo que el de la competencia.

\section{Verificar}

En este paso se considera un periodo de prueba para verificar si las acciones realizadas tienen un correcto funcionamiento, en que se hará seguimiento de las acciones implementadas en el plan de mejoramiento continuo; si la mejora no cumple las expectativas iniciales, habrá que modificarla para que se ajuste a los objetivos esperados.

En la primera etapa se espera los siguientes resultados: $10 \%$ menos en costos operativos, $8 \%$ de incremento en la productividad y $20 \%$ menos tiempo en operación de planta. En la segunda etapa: 25\% menos en costos operativos, 20\% de incremento en la productividad y $25 \%$ menos tiempo en operación de planta. Finalmente, en la tercera etapa se espera: $35 \%$ menos en costos operativos, $25 \%$ de incremento en la productividad y $30 \%$ menos tiempo en operación de planta.

\section{Matriz de auto-calidad}

Esta matriz se utiliza para hacer seguimiento a la implementación del plan de mejoramiento continuo. $\mathrm{El}$ proceso de implantación se constituye en cinco fases: formación e implicación de los empleados, selección de las áreas para el desarrollo de la Matriz de auto-calidad MAQ, puesta en marcha de la MAQ, utilización e integración de la MAQ en el trabajo diario, aplicación y análisis de resultados.

\section{Resultados esperados}

Reducción de costos operativos. disminuyen los costos de los recursos. El proceso de explotación de yeso es eficiente y se reduce la ocurrencia de errores y demoras. Se emplea mejor el tiempo de uso de maquinaria y materiales. Incremento de la productividad, como consecuencia de la reducción de costos directos e indirectos. Mejor control, para solucionar problemas de manera oportuna. Trabajo en equipo, los resultados anotados en la MAQ exigen colaboración y trabajo en equipo. Implicación de los trabajadores, gracias al trabajo en equipo y a la asimilación de las metas empresariales como propias.

\section{Actual}

Para determinar el ciclo se deben estudiar los resultados desde el conocimiento (know how): ¿Qué se aprendió? Se debe utilizar la información disponible que ofrece el Sistema ERP para mejorar los procesos, como ser: reducción de costos y optimización en los procesos de producción. ¿Dónde más podría ser aplicado? Al ser procesos similares este plan de mejoramiento continuo puede ser aplicado a todos los procesos de explotación de la empresa en todas las canteras y materiales. ¿De qué manera puede ser estandarizado? Al realizar la secuencia de las 
etapas planteadas en el plan de mejoramiento continuo para los otros materiales y canteras. ¿Cómo mantener la mejora lograda? Manteniendo el método Deming $y$ el mejoramiento continuo como parte de la política empresarial.

Una vez finalizado el proceso se estudian los resultados y se determina si se tiene un proceso de explotación de materias primas eficiente que presente menores costos. Si se cumplen los objetivos planteados; si los resultados no son satisfactorios, se deberán realizar los respectivos cambios para ajustar los resultados al objetivo. En este punto se concluye el ciclo, pero por tratarse de un proceso de mejora continua se debe pensar que existen objetivos superiores, por lo que una vez terminado este paso se debe volver a iniciar el ciclo.

\section{CONCLUSIONES}

El Sistema ERP ha sido adecuado al tamaño y necesidad de la empresa. Después de tres años de su implementación, los beneficios son tangibles y son valorados por los funcionados; existen mejoras en los módulos y los procesos son más rápidos. Destacan la reducción de tiempos operativos, eliminación de procesos repetitivos, mejor planificación de los recursos, visibilidad de la información en toda la empresa y un aumento en la capacidad de procesamiento de la información. La situación financiera de la empresa presenta deficiencia en el control de costos, no se diferencias los costos por proceso ni por producto.

En el análisis histórico de ratios financieros se observó que desde la implementación del Sistema ERP aumentó la liquidez de la empresa, se debe principalmente, a contar con información precisa que ayuda a tomar decisiones y desarrollar estrategias para tener una mejor administración de los recursos. En cuanto a la rotación de inventarios se tiene una tendencia negativa, debido a la falta de control de los costos de minerales vendidos que presentan un comportamiento irregular en las distintas gestiones. En la rotación de cuentas por cobrar se ve una tendencia creciente desde la implementación del Sistema ERP, gracias a que ahora la empresa cuenta un mecanismo de control que le facilita hacer efectivas sus cuentas por cobrar. Al contar con mayor liquidez la Empresa tiene mayor solvencia, ya que tiene la capacidad de pago necesaria para hacer frente a sus obligaciones en los tiempos establecidos. El margen de ganancia bruta presenta una tendencia a ir incrementando si se utiliza de manera eficiente la información generada por el Sistema ERP, lo que se traduce en un mayor beneficio para la empresa y para los accionistas.

La aplicación de método Deming y el mejoramiento continuo permitieron un manejo eficiente de la información generada por el Sistema ERP. Contar con información confiable, clara y oportuna de los distintos procesos de explotación de materias primas es una ventaja para la toma de decisiones.

\section{REFERENCIAS}

Azzarini, A; Blanco G \& Perdomo D. (2011). Factores críticos de éxito en la implementación de ERP en grandes empresas de Uruguay

Deming, W. E., \& Medina, J. N. (1989). Calidad, productividad y competitividad: la salida de la crisis. Ediciones Díaz de Santos

Díaz, A., Gonzales, J. C., \& Ruiz, M. E. (2005). Implantación de un sistema ERP en una organización. RISI, 2(3), 30-37

Gitlow, H. S., \& Gitlow, S. J. (1989). Cómo Mejorar La Calidad y la Productividad con el Método Deming: una guía práctica para mejorar su posición competitiva. Norma 
Knouse, S. B., Carson, P. P., Carson, K. D., \& Heady, R. B. (2009). Improve constantly and forever: The influence of W. Edwards Deming into the twenty-first century. The TQM journal

Pinto, S. M., Ramírez, P. E., \& Grandón, E. E. (2017). Antecedentes del Éxito de los sistemas de planificación de recursos empresariales en las grandes empresas chilenas: Un modelo factorial exploratorio. Información tecnológica, 28(3), 139-146

Pollock, N., \& Williams, R. (2008). Software and organisations: The biography of the enterprise-wide system or how SAP conquered the world. Routledge

Uwizeyemungu, S., \& Raymond, L. (2010). Linking the effects of ERP to organizational performance: Development and initial validation of an evaluation method. Information Systems Management, 27(1), 25-41

Vera, Á. B. (2006). Implementación de sistemas ERP, su impacto en la gestión de la empresa e integración con otras TIC. Capic Review, (4), 3

Walton, M. (1988). Cómo administrar con el método Deming (No. 658.04 W222c). Editorial Norma 